\title{
Introduction to the Social \& Psychological Perspectives in Collaboration Research Minitrack
}

\author{
GJ de Vreede, Triparna de Vreede, Paul Spector \\ University of South Florida \\ gdevreede@usf.edu,tdevreede@usf.edu,pspector@usf.edu
}

Technology supported collaboration and communication between individuals entails complex social and psychological situations. An understanding of social and psychological aspects of collaboration is essential to creating productive work environments. The use of collaboration and communication systems is framed by the psychological and social factors concerning the users and their work environment. It is important to understand these factors to successfully facilitate the sustained use of these technologies. Further, knowledge of the psychologicalsociological aspects of technology-supported collaboration and communication also assists in detecting, avoiding, and effectively resolving issues that may arise from using such technologies.

Since its inception in 2012 at HICSS-45, this minitrack has provided a venue for studying issues related to the dynamic interplay between people, their environment, and the collaboration technologies they use to create collaborative value. It has hosted research papers and presentations that addressed a variety of topics and theoretical perspective. Examples include, but are not limited to personality, cultural psychology, social psychology, cognitive psychology, diversity, leadership, prejudice and discrimination, attitudes and social intelligence, social learning theory, self efficacy, behavioral theories, violence and aggression, attractions and affiliations in groups, and group psychology.
Over the years, the minitrack has attracted attendees from business schools, IS researchers with a psychological/sociological focus, industrial/organizational psychologists, and management researchers. At HICSS-46 a paper from this minitrack was honored as the best paper in the Collaboration Systems and Technologies Track.

While the earliest minitracks focused mainly on organizational contexts of technology use, later minitracks have shown an increasing focus on issues surrounding the use of social media by individuals, teams, and organizations. This focus is likely to remain dominant for the coming years as social media applications and technologies can still be considered in their formative phase yet are unlikely to fade away any time soon.

This year's minitrack features four papers that address a variety of topics:

- Am I a Leader? Examining Incongruence in the Leader Identity Construction Process, by Amy Bartels

- The Optimal Experience: Social Identity and IT Identity as Antecedents of Group Flow in Social Media Use, by Eric Curley and Greta Polites

- Influence of Culture on Reactions to Negotiation Deadline, by Zhaleh Semnani-Azad, Wendi Adair, Katia Sycara, and Michael Lewis

- LeadLets: Towards a Pattern Language for Leadership Development of Human and AI Agents, by Triparna de Vreede, Logan Steele, Gert-Jan de Vreede, and Robert Briggs. 